
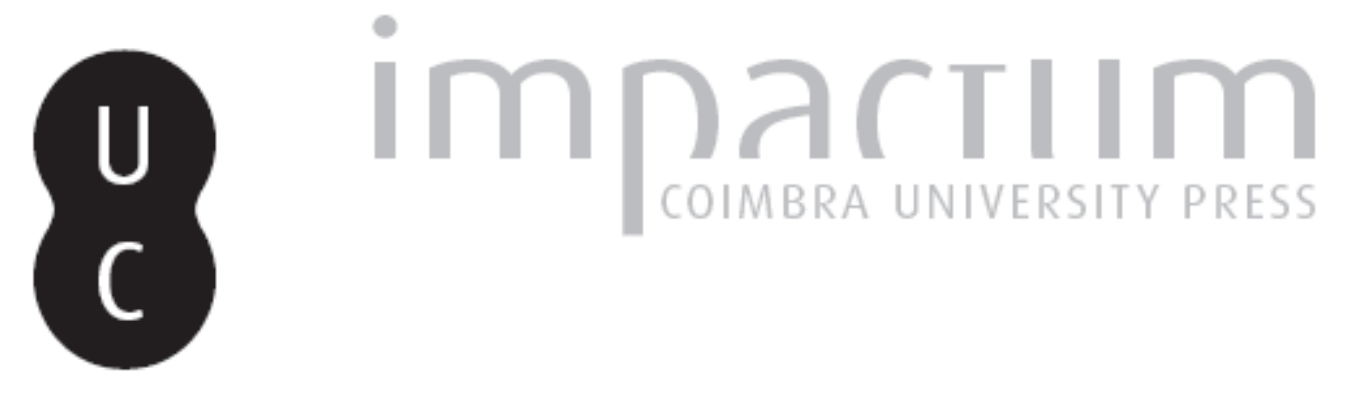

\title{
Conservación e investigación en el área de Antropología Biológica del Museo Etnográfico J. B. Ambrosetti (Facultad de Filosofía y Letras, Universidad de Buenos Aires, Argentina)
}
Autor(es):
Aranda, Claudia; Luna, Leandro; Costanzo, Natalia; Contissa, Valeria;

Publicado por: Imprensa da Universidade de Coimbra

URL persistente:

URl:http://hdl.handle.net/10316.2/39074

DOI:

DOI:http://dx.doi.org/10.14195/2182-7982_31_1

Accessed : $\quad$ 26-Apr-2023 14:53:40

A navegação consulta e descarregamento dos títulos inseridos nas Bibliotecas Digitais UC Digitalis, UC Pombalina e UC Impactum, pressupõem a aceitação plena e sem reservas dos Termos e Condições de Uso destas Bibliotecas Digitais, disponíveis em https://digitalis.uc.pt/pt-pt/termos.

Conforme exposto nos referidos Termos e Condições de Uso, o descarregamento de títulos de acesso restrito requer uma licença válida de autorização devendo o utilizador aceder ao(s) documento(s) a partir de um endereço de IP da instituição detentora da supramencionada licença.

Ao utilizador é apenas permitido o descarregamento para uso pessoal, pelo que o emprego do(s) título(s) descarregado(s) para outro fim, designadamente comercial, carece de autorização do respetivo autor ou editor da obra.

Na medida em que todas as obras da UC Digitalis se encontram protegidas pelo Código do Direito de Autor e Direitos Conexos e demais legislação aplicável, toda a cópia, parcial ou total, deste documento, nos casos em que é legalmente admitida, deverá conter ou fazer-se acompanhar por este aviso.

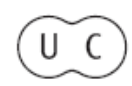




$$
\bar{A}
$$




\section{Conservación e investigación en el área de Antropología Biológica del Museo Etnográfico J. B. Ambrosetti (Facultad de Filosofía y Letras, Universidad de Buenos Aires, Argentina)}

\section{Claudia Aranda', Leandro Luna ${ }^{2}$, Natalia Costanzo ${ }^{3}$, Valeria Contissa ${ }^{3}$, Natalia Gámez ${ }^{3}$, Paula Godoy, Karina Zuccala ${ }^{4}$}

arandaclau@gmail.com, lunaranda@gmail.com

DOI: http://dx.doi.org/10.14195/2182-7982_31_1

Resumen Se detalla parte de las actividades realizadas durante el año 2012 en el marco de un proyecto de conservación en el Área de Antropología Biológica del Museo Etnográfico J. B. Ambrosetti, Universidad de Buenos Aires, Argentina. Este Plan Integral de Manejo de Colecciones Osteológicas se propone desarrollar un protocolo basado en el tratamiento ético de los restos

\begin{abstract}
This paper details part of the activities about conservation of human remains carried out during 2012 in the Area of Biological Anthropology (Ethnographic Museum J. B. Ambrosetti, University of Buenos Aires, Argentina). This Integral Plan of Management of Osteological Collections aims to develop a protocol based on the ethical treatment of human remains, highlighting the concepts of custody,
\end{abstract}

\footnotetext{
1 Jefa del Área de Antropología Biológica, Museo Etnográfico J. B. Ambrosetti, Facultad de Filosofía y Letras, Universidad de Buenos Aires, Buenos Aires, Argentina.

${ }^{2}$ CONICET. Museo Etnográfico J. B. Ambrosetti, Facultad de Filosofía y Letras, Universidad de Buenos Aires., Argentina.

${ }^{3}$ Estudiante de la Lic. en Conservación y Restauración de Bienes Culturales, Universidad del Museo Social Argentino, Buenos Aires, Argentina.

${ }^{4}$ Área de Antropología Biológica, Museo Etnográfico J. B. Ambrosetti, Facultad de Filosofía y Letras, Universidad de Buenos Aires, Buenos Aires, Argentina.
} 
humanos, destacándose los conceptos de custodia, identidad y respeto, que cubre aspectos articulados en cuatro ejes principales: ética, educación/investigación, conservación y patrimonio. Dentro de ese marco, se discuten los procedimientos específicos implementados tendientes a la salvaguarda y minimización del deterioro de un conjunto de restos humanos afectados por agentes medioambientales. Se enfatiza en la interacción entre especialistas de diferentes disciplinas para desarrollar tareas de conservación desde una perspectiva integradora. Esta forma de abordar las colecciones establece nuevas estrategias que contemplan la necesidad de desarrollar tareas de conservación y analizar la información científica en un marco que priorice los aspectos bioéticos de las políticas de manejo patrimonial. Los resultados son prometedores para la caracterización de las colecciones, lo cual incluye la identificación, documentación, conservación y guarda de restos humanos prehistóricos procedentes de diferentes regiones del mundo. La aplicación de esta propuesta surge desde la bioantropología para abordar las investigaciones de restos humanos desde una perspectiva integral. Se pretende contribuir a generar conciencia entre los investigadores de restos humanos sobre la importancia de su participación en la generación de protocolos de conservación.

Palabras clave: Plan de manejo; restos humanos; investigación; interdisciplina; bioética.

identity and respect. It covers several aspects that are divided into four main areas: ethics, education/research, conservation and heritage. Within this framework, the specific procedures developed to safeguard and minimize the deterioration of a set of human remains affected by environmental agents are discussed. The interaction between students from different disciplines in order to implement conservation tasks from an integrative perspective is emphasized. This approach provides new strategies that include the need to develop detailed conservation procedures and to analyze scientific information in a framework that prioritizes the bioethical aspects of management policies. The results obtained are promising for the characterization of the collections housed in the area, including the identification, documentation, preservation and warehousing of prehistoric human remains from different regions of the world. The implementation of this proposal is an example of the approach that comes from bioanthropology to address human remains investigations from a holistic perspective. It is intended to contribute to raising awareness among researchers interested in the analysis of human remains about the importance of their participation in the generation of conservation protocols.

Keywords: Management plan; human remains; investigation; interdisciplinarity; bioethics. 


\section{Introducción}

El Área de Antropología Biológica del Museo Etnográfico J. B. Ambrosetti (Facultad de Filosofía y Letras, Universidad de Buenos Aires, Buenos Aires, Argentina) alberga más de 14.000 elementos óseos y dentales, así como también numerosos restos momificados, representantes de grupos nativos que proceden de diferentes zonas geográficas de Argentina y de otros países, lo que lo convierte en el repositorio de restos humanos más importantes del país y uno de los más destacados de Sudamérica. Entre los objetivos principales de las actividades que allí se realizan se destacan su preservación, diagnóstico osteológico y del estado de preservación, documentación y guarda, así como también la generación de contextos adecuados que promuevan la investigación desde diferentes líneas de análisis y la formación de recursos humanos especializados en la temática. El Área recibe anualmente a gran cantidad de investigadores locales y de diferentes partes del mundo, lo que contribuye fuertemente al desarrollo de las investigaciones bioantropológicas. Estos análisis permiten mejorar el conocimiento de numerosos aspectos biológicos y culturales de las poblaciones que habitaron el extremo suramericano (por ejemplo dieta, movilidad, costumbres funerarias, parentesco, etc.).
A mediados del año 2006, y a raíz del nombramiento de una de las autoras (C. A.) como encargada de las colecciones osteológicas, se redactó y propuso a la Dirección de la institución un Plan Integral de Manejo de Colecciones Osteológicas. Esta propuesta fue motivada principalmente por el alto grado de descontextualización y mezcla de los restos y la ausencia de un entorno protector para las colecciones en ese momento. El proyecto general implementado tuvo por objetivo desarrollar un protocolo basado en el manejo respetuoso de los restos humanos, destacándose los conceptos de custodia, identidad social y compromiso profesional. Dicho plan de trabajo consta de cuatro ejes principales y fundamentales que se articulan entre sí: ética, educación/investigación, conservación y patrimonio. Se puso énfasis desde los inicios de las actividades en priorizar la interacción interdisciplinar, involucrando especialistas que pudieran contribuir al desarrollo del plan desde una perspectiva integradora (Aranda, 2009; Aranda et al., 2010). Afortunadamente, esta propuesta está comenzando a ser repetida en otros repositorios bioantropológicos del país por parte de encargados especialistas que comparten la necesidad de otorgar a las colecciones de un ambiente que priorice en primer lugar el tratamiento respetuoso y en segundo lugar la minimización del deterioro (ver Aranda y Del Papa, 2009). 
Enmarcándose en este enfoque general, fue necesario desarrollar durante el año 2012 un plan más específico e inmediato como consecuencia de una situación de salvataje por problemas de in-

4 fraestructura y anegamiento de parte de la colección. Se presentan en esta oportunidad las actividades desarrolladas y sus implicancias prácticas derivadas, las cuales priorizaron en el relevamiento, diagnóstico general y conservación de los conjuntos osteológicos contenidos en un conjunto de cajas de madera, para lo cual se diseñó un plan para afrontar los problemas específicos derivados de este tipo de inclemencias ambientales. Se subraya que el protocolo de trabajo fue en este caso delineado y desarrollado entre especialistas de diferentes disciplinas interesadas en el estudio de colecciones osteológicas, como la bioarqueología, la museología y la conservación. El objetivo específico del mismo fue doble, se desarrolló mediante actividades simultáneas y se distanció notablemente de las prácticas tradicionales generalmente implementadas en el país: por un lado, aplicar un protocolo de conservación preventiva para mitigar los procesos de deterioro de los restos utilizando técnicas y productos no invasivos, junto con el desarrollo de un relevamiento documental exhaustivo, y por otro, generar el primer conjunto de información bioarqueológica sobre los restos humanos afectados desde múltiples líneas de análisis. Ambos aspectos de este proceso que está recién en sus inicios interaccionan continuamente para producir, en el largo plazo, colecciones protegidas del deterioro y asociadas a información contextual y documental confiable y completa, lo cual las hace más visibles y atractivas para su investigación como consecuencia del mejoramiento en el acceso a la información por parte de los investigadores interesados. Por otra parte, la relación sinérgica entre las actividades de conservación e investigación permiten redimensionar constantemente las aplicaciones técnicas hacia las colecciones en función de las preguntas bioarqueológicas y potenciar la aplicación de nuevos métodos de análisis osteológico para estudios futuros.

\section{Antecedentes de conformación de las colec- ciones osteológicas humanas}

El abordaje moderno hacia las colecciones osteológicas se distancia fuertemente de las prácticas tradicionales de su manejo tal como se realizaban desde fines del siglo XIX hasta el pasado reciente a nivel mundial. Históricamente, las excavaciones en estructuras mortuorias se caracterizaron por sus extensas descripciones del ajuar recuperado y breves menciones de los restos humanos asociados. Se tomaba especial cuidado en recuperar cráneos y huesos largos ente- 
ros (i.e. Imbelloni, 1947; Brothwell, 1967), mientras que los restos deteriorados recibían mínima atención y en la mayoría de los casos eran dejados en el terreno (i.e. Moreno, 1874; Ambrosetti, 1907; Debenedetti, 1910; Casanova, 1930). Generalmente se seleccionaban y recuperaban aquellos huesos que permitían resolver los problemas que se formulaban en esa época, como por ejemplo los relativos a la adscripción racial de los individuos en relación con otros grupos americanos y la evaluación de sus diferencias con respecto a otros habitantes de la región de estudio (Constanzó, 1942; Schobinger, 1988). En los depósitos, los huesos pertenecientes a un mismo individuo eran en general separados (afortunadamente, con algunas notorias excepciones), realizándose la agrupación de elementos de un mismo tipo. En el marco de ese proceso, era común que cada unidad identificada fuera inventariada con un número clasificatorio distinto, lo que hizo sumamente dificultosa la reconstrucción esqueletal posterior (Luna, 2008; Baffi, 2009). Se priorizó en la conservación de los cráneos, ya que constituían las porciones anatómicas que mayor potencial interpretativo contenían según las disquisiciones teóricas de ese momento. A su vez, las técnicas implementadas durante alrededor de 100 años, hasta aproximadamente la década de los 80 del siglo pasado, produjeron importantes deterioros en los conjuntos que supuesta- mente buscaban resguardar. Esta forma de organizar los depósitos de los museos deviene de la historia clasificatoria del coleccionismo naturalista que motivó su creación, así como de los intereses y de las preguntas científicas que imperaron durante ese período; ambos procesos diagramaron las estructuras museísticas a lo largo de todo el mundo (Podgorny y Lopes, 2008; Pegoraro, 2009).

Entre las técnicas de conservación más aplicadas en el pasado, algunas de las cuales lamentablemente se mantienen en el presente por desconocimiento de sus aspectos negativos y una general falta de formación de quienes realizan las aplicaciones prácticas, se destacan la inclusión de tensores metálicos para mantener la asociación de determinadas zonas articulares, el envoltorio de conjuntos óseos con papel de diario, la aplicación de algodón en el interior de la zona nasal, la escritura de siglas y textos en la superficie cortical con tintas contaminantes y en sectores del hueso que ofrecen información bioarqueológica relevante, la aplicación de barnices y lacas, el remontaje de fragmentos óseos con adhesivos nocivos y la limpieza agresiva de los restos, lo que promueve el deterioro de los tejidos esqueletales. Al mismo tiempo, no se prestó demasiada atención a mitigar otros aspectos del deterioro, como por ejemplo la pérdida de elementos dentales y el desarrollo de plagas (Baffi, 2009; Aranda et al., 2010). En 
claro contraste con dichas estrategias, las que están siendo aplicadas desde el año 2006 en diferentes muestras osteológicas del Área de Antropología Biológica se integran dentro de nuevas propuestas importancia la necesidad de desarrollar tareas de conservación preventiva y de analizar su información científica para generar actividades de investigación y docencia, todas las cuales deben ser necesariamente abordadas dentro de un que contemplan en un mismo plano de marco que priorice los aspectos bioéticos de las políticas de manejo patrimonial (Aranda, 2009; Aranda et al., 2010). En este marco, se entiende a la conservación preventiva como cualquier medida o acción que tenga como objetivo evitar o minimizar futuros deterioros o pérdidas de los conjuntos museísticos y que se realicen sobre el contexto ambiental, no directamente sobre los restos (de Guichen, 1999). Se trata de un "... elemento importante de la política de los museos y de la protección de las colecciones. A los profesionales de museos les incumbe la responsabilidad fundamental de crear y mantener un entorno protector para las colecciones almacenadas, expuestas o en tránsito, de las que están encargados" (ICOM, 2006: 5-6; ver Cassman, 1989; Rose, 1992; Vaillant Callol et al., 2003).

\section{Ética y legislación}

Una gran cantidad de restos humanos que se hallan alojados en diferentes museos del mundo fueron excavados durante fines del siglo XIX y principios del siglo XX, cuando aún las etnias a las cuales pertenecían no eran consideradas en igualdad de condiciones respecto del resto de los habitantes de su país. En la actualidad, es abundante la doctrina científica sobre el tratamiento ético de los restos humanos, sobre todo en el marco de los pedidos de restitución que se han multiplicado en las últimas décadas, principalmente en América (i.e. Ubelaker y Grant, 1989; Walker, 2000; AAPA, 2003; Walsh-Haney y Lieberman, 2005). Por ello, resulta de fundamental importancia garantizar el correcto diagnóstico, catalogación y guarda de dichos restos. Las asociaciones profesionales de museos comenzaron a considerar en sus estándares éticos a esos restos y a los objetos sagrados como material sensible que merece un trato cuidadoso y respetuoso (ICOM, 2006).

Este tipo de colecciones es considerado actualmente como parte fundamental del patrimonio cultural, tanto por el poder identitario que poseen para la sociedad como por el conocimiento irreemplazable que brindan en el ámbito científico. Por ello, es indispensable establecer políticas planificadas de manejo, resguardo, documentación y sistematización de la información con 
anterioridad a cualquier implementación práctica (Lemp Urzúa et al., 2008; Aranda y Del Papa, 2009; Aranda et al., 2010; 2012; AABA, 2014). Sin embargo, muchas veces se olvida cuáles son los pasos más elementales (pero no por eso menos necesarios) que implican la conformación de una colección. La acumulación de objetos aislados, aun abundante, no resulta suficiente como para clasificarse como una colección, ya que ésta cobra entidad como tal sólo cuando se asocia a cada objeto su documentación; es allí donde radica la importancia de los esfuerzos en lograr adecuadas caracterizaciones de las muestras osteológicas. Por ello, los bioarqueólogos y antropólogos biólogos tienen la responsabilidad de preservar y registrar para la posteridad las muestras contextualizadas de restos humanos, ya que ofrecen información única sobre la historia de la humanidad (Larsen y Walker, 2005; Aranda y Ramundo, 2010; AABA, 2014). Sólo la asociación entre las tareas del arqueólogo (recuperación de información material y contextual adecuada en el campo), del antropólogo biólogo (identificación y caracterización osteológica) y las de conservación (documentación, tratamiento y guarda) desembocan en el desarrollo de planes de manejo de restos osteológicos adecuados.

Las prácticas de conservación están además enmarcadas en un contexto social que las moldea y dentro de un paradigma teórico que las restringe, de manera que las planificaciones requieren constantemente de la mirada interdisciplinar. Es por este motivo que se convierten en aplicaciones históricamente subjetivas que pretenden contribuir a alcanzar determinados objetivos éticos y de investigación, los cuales varían a medida que pasa el tiempo. De esta forma, las planificaciones referidas a los planes de manejo terminan modificando los conjuntos que se busca resguardar de algunas formas no claramente previsibles. El problema de trabajo aquí descripto es un claro ejemplo de esa compleja dinámica: "Continuity and change, participation, power, and ownership are all bound up in the ways in which cultures are created and progress. The effects of these phenomena of cultural change and evolution are manifested clearly in the heritage conservation arena" (Avrami et al., 2000: 9).

La Ley Nacional Argentina Nº 25.743 de Protección del Patrimonio Arqueológico y Paleontológico, promulgada el 25 de junio del año 2003, establece en su artículo $2^{\circ}$ que "forman parte del patrimonio arqueológico las cosas muebles e inmuebles o vestigios de cualquier naturaleza que se encuentren en la superficie, subsuelo o sumergidos en aguas jurisdiccionales, que puedan proporcionar información sobre los grupos socioculturales que habitaron el país desde épocas precolombinas hasta épocas históricas recientes" (énfasis de los autores). Su contenido es acorde con enunciaciones internacionales al respecto. Entre mu- 
chas otras, el Convenio Europeo para la Protección del Patrimonio Arqueológico (1992), firmado em Malta, establece en su Artículo 1 que "se considerarán elementos del patrimonio arqueológico todos

8 los restos y objetos y cualquier otra huella dejada por la humanidad en épocas pasadas que cumplan los siguientes requisitos: a) que su conservación y estudio ayuden a reconstruir la historia de la humanidad y su relación con el entorno natural; y b) que las principales fuentes de información sobre dichos restos y huellas sean la realización de excavaciones o descubrimientos y otros métodos de investigación de la humanidad y su entorno" (énfasis de los autores; ver García Fortes y Flos Travieso, 2008). En consecuencia, dentro del marco de las reglamentaciones nacionales e internacionales, las colecciones osteológicas constituyen un acervo insustituible del patrimonio cultural mundial y se definen por ser un conjunto único, no renovable y no reemplazable en el que se intersectan aspectos biológicos y culturales de la realidad social. Por este motivo, como cualquier mínima alteración restringiría la información disponible sobre los grupos humanos del pasado, resulta indispensable establecer en forma inmediata políticas planificadas de manejo, resguardo, documentación y sistematización de la información con anterioridad a cualquier implementación práctica (Lemp Urzúa et al., 2008; Aranda, 2009; Aranda y Del Papa, 2009).
Debe destacarse que este Plan Integral de Manejo de Colecciones Osteológicas se desarrolla en el marco de las regulaciones vigentes derivadas de leyes patrimoniales relativamente recientes en Argentina. Las implicancias de la Ley Nacional de la República Argentina $N^{\circ}$ 25.517 y del recientemente promulgado Decreto reglamentario 701/10, derivado de ella, enmarcan legalmente los derechos y obligaciones de los diferentes actores sociales potencialmente involucrados en la problemática, entre los que se destacan las agrupaciones indígenas, otros sectores sociales interesados, la comunidad científica y el Estado. Un aspecto de primer orden que se deriva de estas y otras reglamentaciones (i.e. Acuerdo de Vermillion, 1989; Conferencia Mundial de Derechos Humanos, 1993; Declaración de Helsinki, 2008; Código Deontológico para el Estudio, Conservación y Gestión de Restos Humanos de Poblaciones del Pasado de la Asociación de Antropología Biológica Argentina (AABA, 2014) en relación con la ética del estudio de restos humanos) es mantener como premisa fundamental el respeto al ser humano y promover tanto la dignidad de la persona como la confidencialidad de su identidad, considerando las creencias, percepciones y derechos de todos los grupos locales involucrados (AAPA, 2003; Larsen y Walker, 2005; Aranda et al., 2010; Bosio et al., 2012). A su vez, en la esfera de las obligaciones del científico que se espe- 
cializa en el análisis de los restos humanos, se subraya que una responsabilidad ética primordial es registrar para las generaciones por venir toda la información que proveen sobre la historia de la condición humana y propiciar su perduración para generaciones futuras (Larsen y Walker, 2005). A nivel nacional, el Código Deontológico de la AABA manifiesta que el análisis de restos humanos "... es un objetivo legítimo que resulta de interés y beneficio -efectivo o potencial- para toda la humanidad, independientemente de la nacionalidad, origen, extracción social, credo y demás atributos personales o colectivos, y que no puede ser reemplazado - sin costo- por otras aproximaciones a la historia de los grupos humanos en el ámbito de nuestro territorio nacional" (AABA, 2014: 112). Es en el interjuego entre todas estas consideraciones éticas, contemplando cada una de las voces involucradas, que deben diseñarse los planes de trabajo sobre restos humanos, ya que sólo de esa manera se garantiza un tratamiento respetuoso y ético de los restos (Aranda y Ramundo, 2010). De lo contrario, el encapsulamiento de las disciplinas científicas en relación con la problemática social del momento, derivan a largo plazo en un fracaso rotundo de las prácticas profesionales.

\section{Conservación preventiva e investigación: un encuentro necesario}

Como un derivado directo del Plan Integral de Manejo de Colecciones Osteológicas anteriormente descripto, a principios de 2012 se dio inicio a la planificación e implementación de las actividades específicas motivadas por una serie de problemas edilicios sufridos por el Área, ocasionados por efectos medioambientales que derivaron en importantes filtraciones de agua de lluvia y afectaron numerosas cajas de madera que contenían restos humanos esqueletales y dentales, incluidas en los Módulos I, II y VIII del establecimiento (Figura 1). Dichos conjuntos osteológicos fueron recuperados y trasladados a la institución por diferentes investigadores en fechas anteriores a mediados del siglo pasado. Inmediatamente se delineó un programa de emergencia para mitigar en el corto plazo los daños potenciales. Para ello se conformó un equipo multidisciplinario de tareas, compuesto por estudiantes de la carrera de Licenciatura en Conservación y Restauración de Bienes Culturales de la Universidad del Museo Social Argentino (Buenos Aires, Argentina), investigadores especialistas en bioarqueología y personal del Área. El procedimiento básico, previamente implementado en otros conjuntos osteológicos del Área con requerimientos específicos diferentes, consta de los siguientes pasos: 1) 
documentación inicial y fotografía de la caja y de los restos que contiene; 2) ventilación; 3) relevamiento de los números de inventario y chequeo de su inclusión en el catálogo original del Área; 4) conremoción de suciedad y conservación trol de la información de referencia con la contenida en el interior y en el exterior de la caja; 5) archivo del material escrito recuperado dentro de cada caja; 6) toma de muestras en los casos requeridos; 7 ) de los elementos; 8) relevamiento de variables bioantropológicas (cuantificación general, grado de completitud de cada elemento, sexo, edad, lesiones patológicas, deformación craneana, etc.); 9) incorporación de la información recu- perada en una base de datos generada especialmente para estas actividades; 10) acondicionamiento final y guarda (Aranda y Ramundo, 2010; Aranda et al., 2010; 2012). Al momento de producirse las filtraciones de agua, este mismo protocolo estaba siendo aplicado en otro sector del Área, pero, debido a la rápida afectación de las cajas y de los restos incluidos en ellas, se decidió dar un giro en las actividades planificadas y priorizar la implementación inmediata de acciones de salvataje sobre las mismas.

En primer lugar se modificó la ubicación de las cajas para evitar mayores deterioros; inmediatamente se realizó una documentación fotográfica del estado

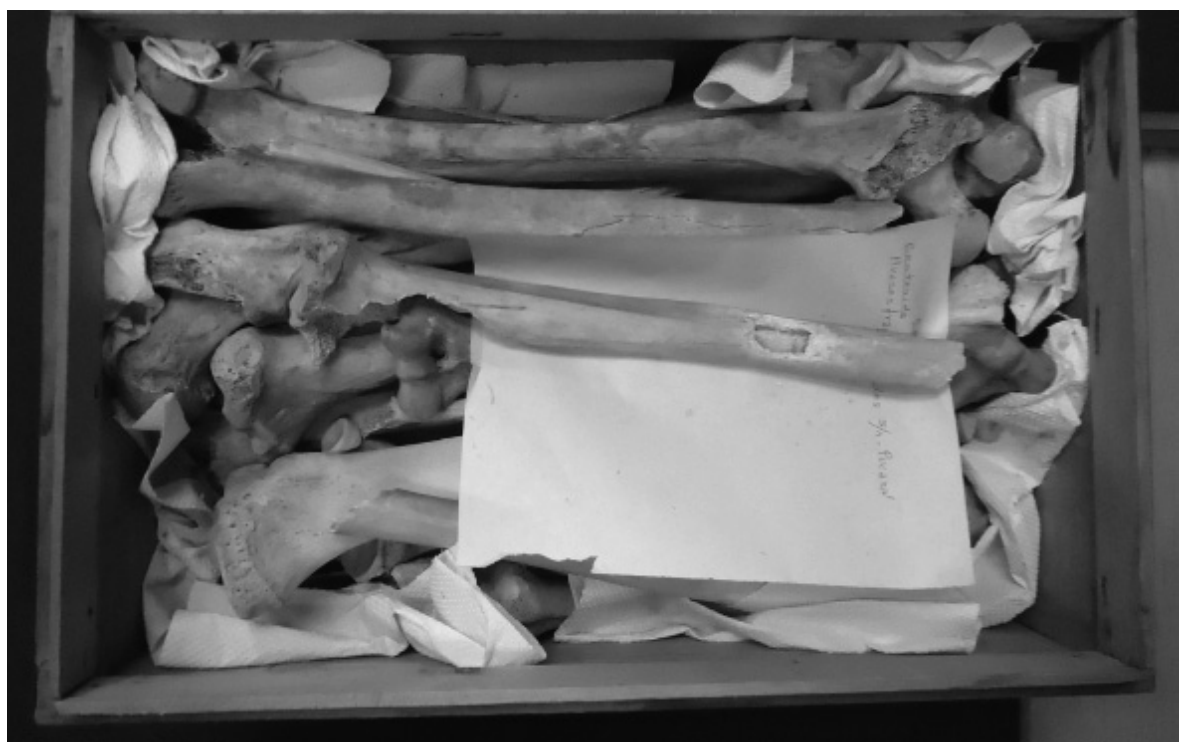

Figura 1. Interior de una de las cajas afectadas. Se observan los efectos del agua en el cortical de varios de los elementos 
de afectación de cada una de ellas, tanto en su estructura como en su contenido. A continuación se efectuó un proceso de secado, tanto de las cajas por un lado como de los restos humanos por otro, mediante un constante control y ventilación (tanto natural como artificial) y evitando la acción directa de los rayos solares. Esta etapa del plan se desarrolló durante un amplio período de tiempo (en algunos casos de hasta dos meses) para asegurar la evaporación del exceso de humedad de los elementos, el cual es uno de los factores de deterioro que más afecta al registro orgánico. En contextos no subacuáticos, este agente potencia el intercambio de oxígeno con el entorno y, en consecuencia, la proliferación de hongos, el deterioro químico y la alteración de la estructura colagénica (Micozzi, 1991; Buikstra y Ubelaker, 1994; Haglund y Sorg, 1997; 2002; González, 2012; Luna et al., 2012).

En paralelo, se implementó una técnica de documentación exhaustiva para relevar toda la información contenida en cada caja. Se registraron los datos presentes en el exterior y en el interior de cada una de ellas y se identificó todo tipo de alteración antrópica en los restos producida desde el momento de la excavación hasta el de guarda (indicios de ataduras en paquetes óseos, tratamientos de remontaje y conservación previos, inclusión de carteles, envoltorios de papel y cualquier otro elemento adicional a los restos óseos, forma de almacenamiento, toma de muestras invasivas, etc.). Este registro exhaustivo de datos posibilita la recuperación de la historia de formación y transformación de cada una de las colecciones osteológicas del Área. También se confeccionó una ficha de relevamiento del estado de preservación de los elementos de cada caja, en la cual se consignó información acerca del nivel y tipo de deterioro, tipo de limpieza efectuada y características de las inscripciones.

Desde el punto de vista estrictamente bioarqueológico, se realizó un relevamiento detallado de los elementos óseos y dentales presentes, indicando tipo de hueso, estado de completitud y fragmentación, agentes tafonómicos que afectaron y relevamiento odontológico general. Se consignó la información disponible sobre el sexo y la edad de los individuos, así como una estimación del número mínimo representado en cada caja (Buikstra y Ubelaker, 1994; Brickley y McKinley, 2004; Luna et al., 2012). En este caso se utilizaron dos fichas de relevamiento, una osteológica para identificar los elementos presentes de cada esqueleto y otra odontológica para describir la ubicación de las diferentes manifestaciones de salud bucal y la preservación dental.

Asimismo, se realizó un control de los números de inventario presentes en cada elemento con la información incluida en los catálogos del área para corroborar la procedencia geográfica y forma de ingreso de cada uno de ellos. Este punto es de 
vital importancia dado que en dichos catálogos no constan en forma sistemática los datos referidos a la ubicación exacta de los restos incluidos en las cajas dentro del Área. Es por ello que la reconstrucción de este tipo de información permitirá a futuro conocer la ubicación de cada elemento en forma rápida y eficiente.

Durante la implementación de este procedimiento se observó la presencia de una gran cantidad de mandíbulas que pertenecerían a una serie de cráneos depositados en otro sector del Área, las cuales han sido separadas en el pasado en concordancia con las propuestas teóricas y los procedimientos instrumentales antaño vigentes. Si bien el contenido de las cajas analizadas no puede ser fácilmente ubicado utilizando el catálogo original, la mayoría de las mandíbulas están rotuladas, lo cual permitió realizar un plan de trabajo de reasignación para devolver la integridad anatómica de los restos craneales, de manera de maximizar la información y disponer de unidades muestrales completas, mejorando sustancialmente las inferencias que pueden generarse actualmente desde una perspectiva bioarqueológica (Aranda y Luna, 2014).

Toda la información obtenida fue incluida en una base de datos en formato Excel, de fácil uso y accesible en el Área para su consulta permanente, de manera de minimizar manipulaciones sucesivas cada vez que un investigador externo solicita acceder a alguna de las colecciones.

De esta manera, el relevamiento exhaustivo de la mayor cantidad de variables en términos descriptivos (i.e. sexo, edad, presencia de manifestaciones patológicas y deformaciones craneanas, alteraciones tafonómicas, efectos de la manipulación antrópica) también contribuye a evitar el deterioro de los conjuntos osteológicos, ofrece una rápida y completa accesibilidad a los datos básicos y mejora la calidad de la información disponible para la transferencia y la atención a los investigadores. Estas actividades de relevamiento contribuyen también a brindar respuestas claras e inmediatas a los pedidos de investigadores interesados en consultar las colecciones, los cuales son cada vez más específicos teniendo en cuenta el constante desarrollo de innovaciones metodológicas y la aplicación de nuevas líneas de trabajo. De esta forma, la decisión de llevar a cabo este relevamiento minucioso no tiene por objetivo principal iniciar estudios bioarqueológicos específicos sino brindar información indispensable para agilizar los procesos administrativos de solicitud de autorización para acceder a las colecciones.

Una vez completamente secos, los restos fueron limpiados mecánicamente mediante la acción de cepillos de diferente dureza, dependiendo de la fragilidad de cada elemento. Esto permitió identificar rasgos biológicos que estaban ocultos por la presencia de depositaciones de sedimento, lo cual aumenta el potencial informativo de estos conjuntos. Finalmente, 
para el sistema de guarda de los restos, se utilizaron materiales aislantes y soportes contenedores dentro de cada caja (Santos Varela, 2002), con el objetivo de amortiguar la acción de distintos agentes deteriorantes, como, por ejemplo, bandejas de espuma de polietileno para evitar la acción de fuerzas físicas directas, y lienciIlo desaprestado para minimizar la absorción de humedad, el depósito de polvo y el acceso de insectos (Figura 2).

\section{Consideraciones finales}

A lo largo de todo el proceso de implementación del plan de trabajo se puntualizó en la interacción sistemática entre conservación e investigación, ya que entendemos que es uno de los aspectos fundamentales en la implementación de protocolos planificados de manejo de colecciones osteológicas. Cada uno de los integrantes del equipo de trabajo recibió saberes procedentes de disciplinas de las cuales no son especialistas, maximizando de esa manera la transferencia de conocimiento, y fue en esa interacción dinámica en la cual todo el proceso alcanzó estándares de calidad mayores a los esperados. En este ámbito, el limitante fundamental no fue el bagaje teórico previo ni las capacidades técnicas sino el mínimo acceso a los insumos materiales necesarios y las inadecuadas condiciones edilicias. En general, los conocimientos y los intereses personales por avanzar en planes integrales de conservación están a la altura de las directrices emanadas por los centros pioneros de la disciplina a nivel mundial,

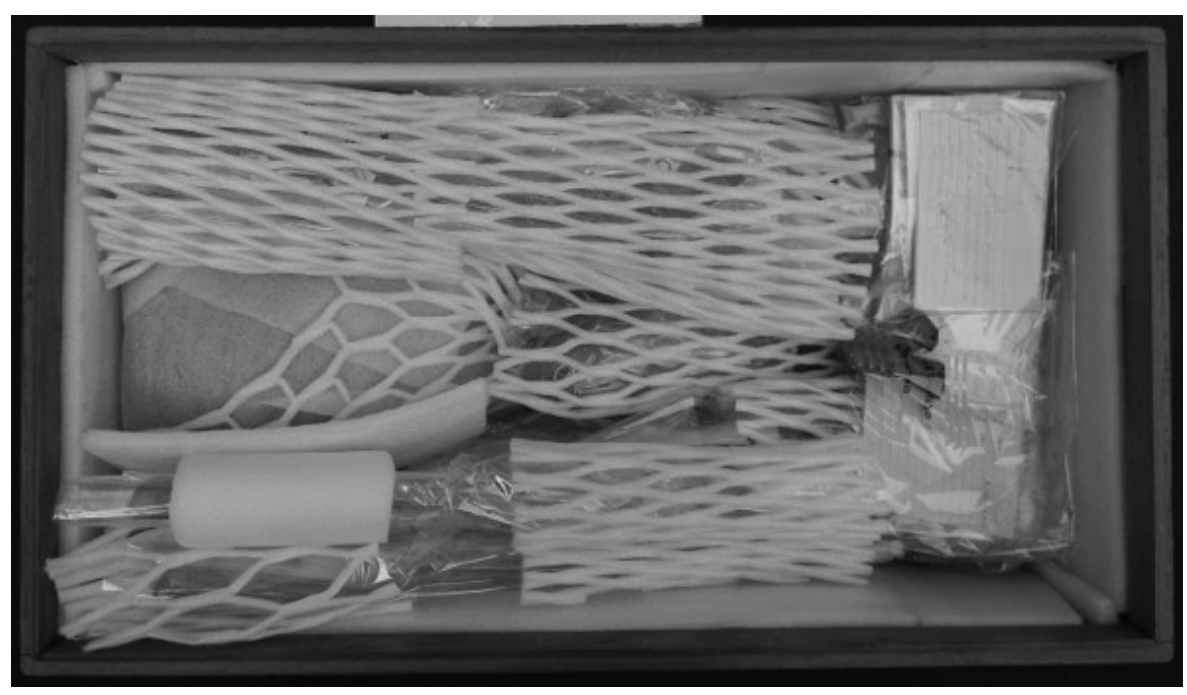

Figura 2. Sistema de guarda final de los restos contenidos en una de las cajas trabajadas 
mientras que las restricciones principales provienen en nuestro país del déficit presupuestario que lamentablemente sufre la mayor parte de las instituciones que albergan colecciones arqueológicas

y etnográficas en Argentina.

En el marco de las conceptualizaciones modernas acerca de las colecciones osteológicas y sus connotaciones éticas, actualmente se considera como imprescindible mantener la asociación de todos los elementos de un mismo individuo junto con la información documental disponible para cada muestra, aplicar estrategias no invasivas de conservación y promover un ambiente protector. Desde esa perspectiva, el Plan Integral de Manejo de Colecciones Osteológicas que se desarrolla desde 2006 en el Área de Antropología Biológica del Museo Etnográfico J. B. Ambrosetti pretende, como uno de sus fines últimos, promover el avance de las investigaciones a través de la generación de información documental completa y detallada, de manera que el potencial interpretativo de estas colecciones pueda estar disponible para investigadores procedentes de cualquier lugar del mundo. Además, a nivel interno, se enmarca en uno de los basamentos básicos que dirige las actividades que se desarrollan diariamente en el Museo Etnográfico, que es considerar que los museos son entes que rescatan, investigan y valorizan la realidad pasada y presente para luego proyectarla de manera crítica a la sociedad (Pérez Gollán y Dujovne, 2001). Dicho plan, que se encuentra en sus primeros pasos y tiene una perspectiva de largo plazo, permitirá desarrollar nuevas investigaciones a partir de las llevadas a cabo en el pasado a partir de una concepción diferente de las muestras osteológicas, potenciándolas como consecuencia de la generación de colecciones más completas y mejor acondicionadas y contextualizadas. Resulta de esencial importancia desarrollar mecanismos para poder mejorar aspectos que hacen a la práctica diaria, centrados en la necesidad de contar con los insumos adecuados y con una infraestructura edilicia que garantice el mantenimiento de un medioambiente adecuado para las colecciones bioantropológicas. Afortunadamente, el principal motor que sostiene el proceso en la actualidad es el espíritu eminentemente vocacional de los recursos humanos formados y en formación en cada una de las especialidades que pueden contribuir a su avance.

\section{Agradecimientos}

A la Dra. Myriam Tarragó, Directora del Museo Etnográfico J. B. Ambrosetti, y a los trabajadores de dicha institución, por el apoyo recibido para realizar las actividades enmarcadas en el Plan Integral de Manejo de Colecciones Osteológicas. 


\section{Referencias bibliográficas}

AABA 2014. Código deontológico para el estudio, conservación y gestión de restos humanos de poblaciones del pasado. Redactado por C. Aranda, G. Barrientos y M. Del Papa (Sub-Comisión de Ética de la Asociación de Antropología Biológica Argentina). Revista Argentina de Antropología Biológica 16(2): 111-113. AAPA. 2003. Code of Ethics. [Online], American Association of Physical Anthropologists. Aprobado el 25 de abril de 2003. [Consultado en 2-3-2013]. Disponible en: http://www.physanth.org/positions/ethics.htm.

Acuerdo de Vermillion sobre Restos Humanos. 1989. World Archaeological Congress (WAC), Vermillion, Dakota del Sur, Estados Unidos.

Ambrosetti, J. 1907. Exploraciones arqueológicas en la ciudad prehistórica de "La Paya". Revista de la Universidad de Buenos Aires, VIII: 5-533.

Aranda, C. M. 2009. Primera etapa del proyecto de manejo de colecciones osteológicas en el Área de Antropología Biológica, Museo Etnográfico J. B. Ambrosetti, Buenos Aires, Argentina. Libro de resúmenes del IV Foro de Conservación del Patrimonio Cultural, Caracas (Venezuela), 9-12 de febrero de 2009. Caracas, Venezuela, Instituto de Estudios Avanzados: 28-29.

Aranda, C. M.; Del Papa, M. 2009. Avances en las prácticas de conservación y mane- jo de restos humanos en Argentina. Revista Argentina de Antropología Biológica, 11(1): 89-93.

Aranda, C; Luna, L. 2014. Inventario y reasociación de restos humanos en el Área de Antropología Biológica del Museo Etnográfico J. B. Ambrosetti (Universidad de Buenos Aires, Argentina). Implicancias para el tratamiento respetuoso de las colecciones y para las investigaciones bioarqueológicas. In: Luna, L.; Aranda, C; Suby, J. (eds.) Avances Recientes de la Bioarqueología Latinoamericana. Buenos Aires, Grupo de Investigación en Bioarqueología: 337-357.

Aranda, C. M.; Luna, L. H.; Ramundo, P. 2012. Primeros análisis y conservación preventiva del registro bioarqueológico del Pukara de la Cueva (Humahuaca, Jujuy). Revista Argentina de Antropología Biológica, 14(2): 9-21.

Aranda, C. M.; Ramundo, P. 2010. Conservación preventiva y protección patrimonial de los restos bioarqueológicos del Pukara de La Cueva (Departamento de Humahuaca, provincia de Jujuy). In: Bárcena, J. R.; Chiavazza, $\mathrm{H}$. (eds.) Arqueología Argentina en el Bicentenario de la Revolución de Mayo. XVII Congreso Nacional de Arqueología Argentina, Mendoza (Argentina), 11-15 de octubre de 2010. Mendoza, Argentina, Facultad de Filosofía y Letras, Universidad Nacional de Cuyo I: 221-226.

Aranda, C. M.; Zuccala, K.; Avido, D.; Salvarredi, A.; Luna, L. H.; Gigliotti, M. V. 2010. Ma- 
nejo de colecciones osteológicas del Museo Etnográfico J. B. Ambrosetti (FFyL, UBA). Actas del $10^{\circ}$ Congreso Nacional de Museos Universitarios, La Plata (Argentina), 20-23 octubre de 2010.

Avrami, E.; Mason, R; de la Torre, M. 2000. Values and heritage conservation. Research report. Los Angeles, The Getty Conservation Institute.

Baffi, E. I. 2009. La vida antes del PVAc. Apuntes para la historia de la conservación en Argentina. Revista Argentina de Antropología Biológica, 11(1): 125-132.

Bosio, L. A.; Garcia Guraieb, S.; Luna, L. H.; Aranda, C. M. 2012. Chacarita Project: Conformation and analysis of a modern and documented human osteological collection from Buenos Aires City. Theoretical, methodological and ethical aspects. HOMO - Journal of Comparative Human Biology, 63: 481-492.

Brickley M.; McKinley, J. 2004. Guidelines to the standards for recording human remains. IFA Paper No. 7 BABAO. Southampton, Department of Archaeology, University of Southampton.

Brothwell, D. 1967. The evidence for neoplasms. In: Brothwell, D.; Sandison, A. (eds.) Diseases in antiquity. Nueva York, Ch. C. Thomas: 320-345.

Buikstra, J.; Ubelaker, D. 1994. Standards for data collection from human skeletal remains. Faytteville, Arkansas, Arkansas Archaeological Survey Research Series No. 44.
Casanova, E. 1930. Hallazgos arqueológicos en el cementerio indígena de Huiliche, Departamento de Belén (provincia de Catamarca). Archivos del Museo Etnográfico, 3: 1-147.

Cassman, V. 1989. Simbiosis entre la arqueología, conservación y museos. Revista Chungara, 23: 93-109.

Conferencia Mundial de Derechos Humanos. 1993. Informe del Director General. Viena. Ms.

Constanzó, M. 1942. Antropología Calchaquí. La colección Zavaleta del Museo Argentino de Ciencias Naturales "Bernardino Rivadavia". Revista del Instituto de Antropología de la Universidad Nacional de Tucumán, 2(9): 213-308.

Convenio Europeo sobre la protección del Patrimonio Arqueológico. 1992. La Valetta, Malta, 16 de enero.

de Guichen, G. 1999. Preventive conservation: a mere fad or far-reaching change? Nueva York, Blackwell Publishers.

Debenedetti, S. 1910. Exploración arqueológica en los cementerios prehistóricos de la Isla de Tilcara (Quebrada de Humahuaca, Provincia de Jujuy). Publicaciones de la Sección Antropológica, 6: 3-263. Buenos Aires, Imprenta y Casa Editorial "Juan A. Alsina".

Declaración de Helsinki de la Asociación Médica Mundial. 2008. ARBOR Ciencia, Pensamiento y Cultura, CLXXXIV: 349-352.

Decreto 701/2010. Comunidades indígenas. Establece que el Instituto Nacional de Asuntos Indígenas será el encargado 
de coordinar, articular y asistir en el seguimiento y estudio del cumplimiento de las directivas y acciones dispuestas por la Ley 25.517. Boletín Oficial del 21/05/10. Buenos Aires, Argentina.

García Fortes, S.; Flos Travieso, N. 2008. Conservación y restauración de bienes arqueológicos. Madrid, Editorial Síntesis.

González, M. 2012. Procesos de formación en el registro bioarqueológico de la subregión Pampa Húmeda y Área Ecotonal Pampa-Patagonia. Tesis Doctoral inédita, Facultad de Ciencias Sociales de la Universidad del Centro de la Provincia de Buenos Aires, Olavarría, Buenos Aires, Argentina.

Haglund, W.; Sorg, M. 1997. Forensic taphonomy: The postmortem fate of human remains. Boca Raton, CRC Press.

Haglund, W.; Sorg, M. 2002. Advances in forensic taphonomy: method, theory, and archaeological perspectives. Boca Raton, CRC Press.

ICOM. 2006. ICOM Code of Ethics for Museums. [Online]. Paris, International Council of Museums. [Consultado en 25-2-2013]. Disponible en http://icom.museum/ code2006 eng.pdf.

Imbelloni, J. 1947. El poblamiento de América. Revista de la Universidad de Buenos Aires, Cuarta época I(1-4): 9-35.

Larsen, C.; Walker, P. 2005. The ethics of bioarchaeology. In: Turner, T. (ed.) Biological anthropology and ethics. From repatriation to genetic identity. Albany, State University of New York Press: 111-120.
Lemp Urzúa, C.; Rodríguez Balboa, M.; Retamal Yermani, E.; Aspillaga Lafontaine, E. 2008. Arqueología del depósito: manejo integral de las colecciones bioantropológicas en el Departamento de Antropología de la Universidad de Chile. Conserva, 12: 69-96.

Ley $N^{\circ}$ 25.743. Protección del Patrimonio Arqueológico y Paleontológico. Promulgada el 25 de junio de 2003, con agregados del Decreto Reglamentario 1022/2004. Buenos Aires, Argentina.

Luna L. 2008. Estructura demográfica, estilo de vida y relaciones biológicas de cazadores-recolectores en un ambiente de desierto. Sitio Chenque I (Parque Nacional Lihué Calel, provincia de La Pampa). BAR International Series 1886. Oxford, Archaeopress.

Luna, L. H.; Aranda, C. M.; García Guraieb, S.; Kullock, T.; Salvarredy, A.; Pappalardo, R.; Miranda, P.; Noriega, H. 2012. Factores de preservación diferencial de restos óseos humanos contemporáneos de la "Colección Chacarita" (Ciudad Autónoma de Buenos Aires, Argentina). Revista Argentina de Antropología Biológica, 14(2): 53-67.

Micozzi, M. 1991. Postmortem change in human and animal remains. A systematic approach. Springfield, Illinois, Charles C. Thomas.

Moreno, F. 1874. Description des cimitieres et paraderos prehistoriques de Patagonie. Revue d'Anthropologie, 3: 72-90. 
Pegoraro, A. 2009. Las colecciones del Museo Etnográfico de la Universidad de Buenos Aires: un episodio en la historia del americanismo en la Argentina, 18901927. Tesis Doctoral inédita, Facultad

Pérez Gollán, J.; Dujovne, M. 2001. De lo hegemónico a lo plural: un museo universitario de antropología. Entrepasados, X(20-21): 197-208.

Podgorny, I.; Lopes, M. 2008. El desierto en una vitrina. Museos e historia natural en la Argentina, 1810-1890. México, Limusa.

Rose, C. 1992. La conservación preventiva. Boletín de APOYO, 3(2). Asociación para la Conservación del Patrimonio Cultural de las Américas.

Santos Varela, M. 2002. Conservación preventiva de una colección única en el mundo: cuerpos momificados Chinchorro. Conserva, 6: 75-86.

Schobinger, J. 1988. Prehistoria de Sudamérica. Culturas Precerámicas. Madrid, Alianza Editorial.

Ubelaker, D.; Grant, L. 1989. Human skeletal remains: preservation or reburial? Yearbook of Physical Anthropology, 32: 249-287.

Vaillant Callol, M.; Doménec Carbó, M. T.; Valentín Rodrigo, N. 2003. Una mirada hacia la conservación preventiva del $\mathrm{Pa}$ trimonio Cultural. Valencia, Editorial de la Universidad Politécnica de Valencia. Walker, P. 2000. Bioarchaeological ethics: a historical perspective on the value of human remains. In: Katzemberg, M.; Saunders, S. (eds.) Biological anthropology of the human skeleton. Nueva York, Wiley-Liss: 3-39.

Walsh-Haney, H.; Lieberman, L. 2005. Ethical concerns in forensic anthropology. In: Turner, T. (ed.) Biological anthropology and ethics. From repatriation to genetic identity. Nueva York, State University of New York Press: 121-131. 